\title{
Stop Mare Mortum y el movimiento de solidaridad con las personas refugiadas en Barcelona
}

\author{
Stop Mare Mortum and the movement of solidarity with \\ refugees in Barcelona
}

\author{
JaVier AlCAlde \\ Universidad Oberta de Catalunya \\ Universidad Autónoma de Barcelona \\ javier.alcalde@uab.cat (ESPAÑA) \\ Martín Portos \\ Universidad Carlos III de Madrid \\ mportos@clio.uc3m.es (ESPAÑA)
}

Recibido: 01.12. 2020

Aceptado: 23.08 .2021

\section{RESUMEN}

Durante el largo verano migratorio de 2015 aumentaba de un modo dramático el nivel de conciencia ciudadana y activismo en Barcelona. En la primavera de 2016, cada día tenían lugar eventos de protesta en solidaridad con las personas refugiadas, promovidos por un amplio espectro de grupos locales, asociaciones y redes. En tanto, un cambio en el gobierno local erigía a una otrora activista social como alcaldesa, asumiendo el tema de las personas refugiadas como una prioridad política. Basado en una serie de entrevistas en profundidad con activistas clave, este artículo presenta, mapea y estudia la evolución de las redes activistas locales. Buscando arrojar luz sobre las dinámicas de mesomovilización, analizamos la plataforma Stop Mare Mortum (SMM). Con un alto nivel de politización y centrándose en las personas refugiadas en tránsito, esta iniciativa nacida de una pequeña red de círculos activistas creció hasta convertirse en una plataforma paraguas con gran capacidad para coordinar iniciativas de la sociedad civil. Junto con una combinación única de emociones y marcos de movilización, la habilidad de SMM para adaptar sus estrategias, repertorios de 
acción y estructuras organizativas a un contexto cambiante explican su capacidad de movilización y el carácter transversal de sus bases.

\title{
PALABRAS CLAVE
}

Refugiados; movimientos sociales; Barcelona; España; Stop Mare Mortum.

\begin{abstract}
The 2015 long summer of migration has increased dramatically the level of citizen awareness and activism in Barcelona. In Spring 2016 a number of protest events in solidarity with refugees were taking place on a daily basis, promoted by a broad range of local groups, associations and networks. In the meantime, a change of government brought a social activist as the new mayor of the city, with the refugees' issue as a top political priority. Based on a number of in-depth interviews with key activists, this article presents, maps and studies the evolution of the local networks. Aiming at shedding light on meso-level mobilization dynamics, we zoom into Stop Mare Mortum. With a high level of politicization and focusing on refugees in transit, this initiative borne out of a small network of activists has gradually become an umbrella platform aiming to coordinate civil society initiatives within this field. Together with a unique combination emotions and frames for mobilization, SMM's ability to adapt its strategies, repertoires of action and organizational structures to a changing environment explains its mobilization capacity and the cross-cut nature of its constituency.
\end{abstract}

\section{KEY WORDS}

Refugees; social movements; Barcelona; Spain; Stop Mare Mortum.

\section{INTRODUCCIÓN ${ }^{1}$}

Según los datos de ACNUR, más de 60 millones de personas se vieron forzadas a huir de sus casas en 2015 (CEAR 2015). En septiembre de 2015 los Estados Miembros de la Unión Europea se comprometieron a acoger a 160.000

${ }^{1}$ Los materiales empíricos de este artículo han sido recabados en el marco del proyecto europeo "Collective Action and the Refugee Crisis", dentro de la ERC Advanced Grant Project "Mobilizing for Democracy. Democratization processes and the mobilization of civil society" (No. 269136), dirigida por D. della Porta. Véase http://cosmos.sns.it/collective-action-and-the-refugeecrisis/. Este artículo expande el trabajo de Alcalde y Portos (2018). Martín Portos reconoce apoyo del programa CONEX-Plus financiado por la Universidad Carlos III de Madrid y el Programa de investigación e innovación Horizonte 2020 de la Unión Europea bajo el acuerdo Marie Sklodowska-Curie (No. 801538). 
refugiados en un plazo de dos años (Comisión Europea 2015; véase el gráfico sobre la evolución de las demandas de asilo en Arcarons 2016: 108, con datos de Eurostat). Debido a la profundidad, visibilidad mediática e impacto internacional de los flujos de solicitantes de asilo a través de las rutas del Mediterráneo, se trataba de una de las coyunturas más críticas de los últimos años. Solo en 2015, llegaron a Italia y Grecia miles de personas (Zamponi 2017). Por su parte, España forma parte de la zona Schengen, siendo la única frontera con el continente africano; esta situación geoestratégica debería facilitar la llegada de migrantes, sobre todo de aquellos que provienen del continente africano (Alcalde y Portos 2018: 155). Sin embargo, en agudo contraste con otros países europeos, solo el $1 \%$ de las personas que realizaron una petición de asilo en Europa durante 2015 lo hicieron en España (Galarraga 2017). En otras palabras, el país no experimentó una llegada masiva de personas refugiadas provenientes de las rutas del Mediterráneo durante la "crisis de los refugiados" afectó particularmente a los inmigrantes que residen en España y desde 2008 muchos han vuelto a sus países de origen (Bastia 2011; Royo 2014; Hellgren y Serrano 2017).

Exceptuando la "crisis de los cayucos" de 2006 en las Islas Canarias, el número de llegadas a las costas españolas ha sido relativamente bajo, en un contexto de políticas migratorias restrictivas (Zaragoza-Cristiani 2016; Kemp 2016). ${ }^{3}$ De hecho, estas cifras pueden explicarse en parte por las duras condiciones impuestas por el gobierno español y las autoridades marroquíes para prevenir la llegada de personas que cruzan (legal o ilegalmente) la Frontera Sur (véase Alcalde y Portos 2018). Así, los principales factores que explican el reducido número de personas refugiadas que deciden buscar protección en España son las barreras físicas (por ejemplo, las vallas en Ceuta y Melilla), la actitud hostil por parte de la policía, el origen geográfico de los solicitantes de asilo (aquellos que vienen huyendo de las guerras de Oriente Medio a menudo eligen otras rutas más cortas), así como unas expectativas de éxito limitadas para los solicitantes de asilo, obstáculos burocráticos y retrasos en el proceso de decisión (Arcarons 2016).

A pesar de la posición ambigua del gobierno conservador del Partido Popular hacia las personas refugiadas - acogiendo a finales de 2018 solo un 16,6\% de la cuota pactada en 2015 (La Vanguardia 2018)—, durante 2015-2016 surgió un fuerte movimiento de solidaridad en todo el país, que tuvo presencia incluso en otros lugares. Por ejemplo, fueron numerosos los activistas españoles que viajaron a los puntos calientes, como los campos de personas refugiadas en Grecia, mostrando el alto nivel de conciencia de la sociedad civil española hacia este colectivo. ${ }^{4}$ Por su parte, muchas organizaciones de prestigio que trabajan en esta

${ }^{2}$ Para un análisis crítico de este concepto, véase Alcalde (2016).

3 "En agosto de 2014, más de 1200 migrantes sub-saharianos llegaron a las costas españolas en menos de 48 horas. Nunca antes habían llegado tantos migrantes irregulares desde las costas marroquíes en un período tan corto de tiempo. De hecho, el número de inmigrantes que llegaron a España por mar entre el 10 y el 12 de agosto de 2014 fue la mitad del número total que llegaron a las mismas costas en todo 2013" (Zaragoza-Cristiani 2016: 19).

4 Por ejemplo, un activista local de Cádiz comentaba: "hace unos días estuvo aquí el repre- 
área con distinto grado de institucionalización mantuvieron un papel relevante en la fase de recepción y acogida, como la Comisión Española de Ayuda al Refugiado (CEAR) y la Cruz Roja. Además, se crearon por todo el país cientos de iniciativas solidarias a escala local.

En Barcelona cabe destacar a Stop Mare Mortum (SMM), una organización dedicada específicamente al tema de personas refugiadas en tránsito. Siendo un ejemplo poco frecuente de organización que consiguió movilizar a decenas de miles de personas (BBC 2017; The Guardian 2017), este artículo pretende arrojar luz sobre la trayectoria y las características organizativas de SMM, contribuyendo de este modo a una literatura académica aún poco numerosa, pero que ha ido creciendo en los últimos años. A partir de las movilizaciones en solidaridad con los refugiados que tuvieron lugar a la sombra del 'largo verano migratorio' de 2015, algunos estudios han explorado las condiciones y las maneras en las que se llevaron a cabo 'actos de ciudadanía' y actividades de protesta (della Porta 2018). Centrados en el caso español, Alcalde y Portos (2018) han señalado la importancia de unas estructuras de oportunidad política cambiantes a escala local, como los niveles de represión y la existencia de aliados, para explicar la diferencia de fuerza del movimiento social en contextos diferentes. En un artículo reciente, Bazurli (2019) destacaba la interacción entre activistas y decisores políticos, mostrando cómo las iniciativas de 'ciudades de bienvenida' desarrolladas en Milán y Barcelona se encuentran en un contexto multinivel que penaliza a las ciudades en los temas relacionados con la política y la gestión de la migración. Centrándose en la dimensión organizativa, el presente artículo toma un enfoque distinto y explora las condiciones más amplias - repertorios de movilización, marcos y emociones - que facilitan una gran capacidad de movilización política en el contexto urbano al nivel meso (es decir, centrado en una organización o grupo específicos), tradicionalmente poco examinado en los estudios sobre movimientos sociales (Andretta, Piazza y Subirats 2015: 200).

Gracias a su elasticidad a lo largo del tiempo, SMM fue capaz de canalizar los esfuerzos de movilización para abarcar un conjunto transversal de miembros y simpatizantes. Transformó así las interacciones entre los actores sociales en este campo, convirtiéndose en el actor crucial en el debate sobre el asunto de las personas refugiadas a escala local (y no solo). Ello fue posible, y esta es la principal idea que desarrollamos, debido a dos factores relacionados entre sí. Por un lado, existe una combinación única de emociones y marcos para la movilización, fundamental en el surgimiento de SMM. Por otro lado, esta plataforma muestra una destacable capacidad para adaptar sus estrategias, repertorios de acción y estructuras organizativas a un entorno cambiante.

El material empírico utilizado en este texto proviene de veinte entrevistas semiestructuradas con activistas e informantes clave llevadas a cabo en el marco de un reciente proyecto de investigación europeo sobre acción colectiva y la

sentante de ACNUR. Al parecer, en los últimos meses han realizado una campaña de recogida de firmas para una petición. En total han recogido 1,5 millones y de ellas, 500.000 venían de España, de lejos el país con mayor número de firmas" (Entrevista E1). 
crisis de personas refugiadas. Las entrevistas nos han permitido descubrir las experiencias de los activistas y su interpretación de la realidad, así como acceder a un amplio rango de actores relevantes que examinan los significados y el contexto semántico de la movilización y ofrecen "una ventana longitudinal en el activismo de los movimientos sociales" (Blee y Taylor 2002: 92-95).

Antes de decidir la estrategia para reclutar a entrevistados, hemos hecho un estudio en profundidad del caso y un mapeo de los actores activos en el terreno. Del análisis preliminar emergió la necesidad de cubrir una serie de perfilesque se convertirían en pautas para el muestreo de entrevistados-, a saber: 1) deberíamos cubrir actores trabajando en distintos niveles de movilización, desde el más local al nacional; 2) incluir voces y representantes de distintos sectores, como el Ayuntamiento de Barcelona, sindicatos o las asambleas de barrio; 3) dar cuenta de la variedad funcional dentro del movimiento social, incluyendo a personas que trabajan con medios (portavoces), pero también logística, organización, etc.; 4) dar cabida también a la perspectiva de personas que habían sido activas en el exterior, en el terreno. Poniendo en común las perspectivas de los distintos casos de estudio en el seno del proyecto internacional, habida cuenta de las particularidades de cada contexto, hemos pactado seguir la estrategia de la bola de nieve ("snowballing"). La primera entrevista nace de un contacto personal de uno de los actores, trabajadora social con menores no acompañados y participante en varios eventos de SMM, que permitió abrir el campo y progresivamente expandir nuestras redes. De este modo, teniendo en cuenta los criterios mencionados previamente, durante las entrevistas se valoraba con los entrevistados cómo seguir adelante y cuál sería la persona que tenía más sentido entrevistar. Asimismo, y en la medida de lo posible, hemos tratado de equilibrar factores sociodemográficos (sexo, edad) y membresía organizacional. Mientras la veintena de entrevistas realizadas sirve para contextualizar y enmarcar el análisis, en este artículo analizaremos en profundidad nueve de ellas, utilizando algunos extractos para ilustrar nuestros argumentos (véase Tabla 1). 


\begin{tabular}{r|r|r|r|r|r|r}
$\begin{array}{r}\text { Código } \\
\text { entrevista }\end{array}$ & Fecha & Edad & Sexo & Lugar & $\begin{array}{r}\text { Miembro } \\
\text { SMM }\end{array}$ & $\begin{array}{r}\text { Otra organiza- } \\
\text { ción }\end{array}$ \\
\hline E1 & $12 / 10 / 2016$ & 40 & $\mathrm{M}$ & Cádiz & & Asamblea local \\
\hline E2 & $28 / 06 / 2016$ & $50-$ & $\mathrm{M}$ & $\begin{array}{r}\text { Barcelo- } \\
\text { na }\end{array}$ & $\mathrm{x}$ & \\
\hline E3 & $23 / 05 / 2016$ & 25 & $\mathrm{H}$ & $\begin{array}{r}\text { Barcelo- } \\
\text { na }\end{array}$ & & Fotomovimiento \\
\hline E4 & $28 / 06 / 2016$ & 48 & $\mathrm{H}$ & $\begin{array}{r}\text { Barcelo- } \\
\text { na }\end{array}$ & & Asamblea local \\
\hline E5 & $28 / 06 / 2016$ & 65 & $\mathrm{M}$ & $\begin{array}{r}\text { Barcelo- } \\
\text { na }\end{array}$ & $\mathrm{x}$ & \\
\hline E6 & $22 / 05 / 2016$ & 23 & $\mathrm{M}$ & $\begin{array}{r}\text { Barcelo- } \\
\text { na }\end{array}$ & $\mathrm{x}$ & \\
\hline E7 & $28 / 06 / 2016$ & $30-$ & $\mathrm{H}$ & $\begin{array}{r}\text { Barcelo- } \\
\text { na }\end{array}$ & $\mathrm{x}$ & \\
\hline E8 & $27 / 06 / 2016$ & 39 & $\mathrm{M}$ & $\begin{array}{r}\text { Barcelo- } \\
\text { na }\end{array}$ & & Fotomovimiento \\
\hline E9 & $25 / 05 / 2016$ & 52 & $\mathrm{H}$ & $\begin{array}{r}\text { Barcelo- } \\
\text { na }\end{array}$ & $\mathrm{x}$ &
\end{tabular}

Tabla 1. Descripción de las entrevistas utilizadas en el artículo.

Bebiendo de la sociología de los movimientos sociales, este artículo se estructura como sigue. Empezamos presentando el contexto local de nuestro caso de estudio, SMM. Desde una perspectiva micro, ilustramos el origen y la creación de esta plataforma, sus marcos estratégicos y los desarrollos emocionales de los activistas, que son clave para la dinámica de movilización. A continuación, nos centramos en algunos puntos de inflexión y en eventos transformativos en la vida organizativa de SMM, sus repertorios de acción y las interacciones con otros actores relevantes en este sector, especialmente sus aliados y oponentes. Finalmente, concluimos destacando los principales hallazgos de nuestra investigación y señalando algunas líneas de investigación futuras.

\section{EL ORIGEN DE STOP MARE MORTUM}

Cuando el movimiento social de solidaridad con las personas refugiadas florecía en medio de la crisis en el Mediterráneo, las elecciones municipales del 24 de mayo de 2015 situaron a Ada Colau como la nueva alcaldesa de Barcelona. Se trataba de una activista social, ex portavoz de la Plataforma de Afectados por la Hipoteca (PAH). En contraste con los obstáculos que caracterizan a la acción del gobierno español a la hora de acoger a las personas refugiadas, este asunto era una prioridad para la coalición política que lideraba Colau. Así, el Ayuntamiento de Barcelona decidió crear un plan de Barcelona como 'ciudad refugio' 
para preparar a la ciudad de cara a una futura acogida de personas refugiadas. Además, contribuyó a establecer una red de ciudades refugio en España e incluso acabó coordinando la iniciativa europea de 'ciudades solidarias' (García Agustín y Jørgensen 2018). Al mismo tiempo, una amplia gama de grupos, asociaciones y redes se centraban en iniciativas de solidaridad a escala local (por ejemplo, personas que ofrecían sus propias casas para acoger personas refugiadas) y promovían una serie de eventos de protesta. Estas movilizaciones tenían una naturaleza mixta. Por un lado, estaban conectadas a ejemplos previos de activismo, sobre todo (aunque no solo) en las áreas de inmigración y de cooperación al desarrollo. Por otro, incluían a organizaciones y grupos creados en esos meses de 2015 y 2016, muchos de ellos surgidos a partir de pequeñas iniciativas, incluso personales.

Precisamente, el entrevistado E9, que es una persona activa en temas de inmigración desde hace muchos años y con cierta relevancia pública en calidad de periodista, envió a título personal un correo electrónico el domingo 19 de abril de 2015 a unas pocas decenas de personas sensibilizadas con el tema, fundamentalmente miembros de ONG y activistas sociales. Si bien algunos receptores pertenecen a asociaciones y entidades diversas, se les pide que participen en la reunión representándose a sí mismos y que difundan el mensaje entre sus redes de contactos. Reproducimos a continuación parte del email con permiso del emisor, porque nos ayudará a entender algunos rasgos clave en relación con los objetivos estratégicos y organizativos y con los marcos interpretativos de SMM:

Os envío este correo electrónico para convocaros a una primera reunión para discutir la posibilidad de una manifestación o una concentración contra las políticas europeas de fronteras e inmigración. Estas políticas están relacionadas con miles de muertes que han tenido lugar en el Mediterráneo desde hace años. [...] La ausencia de una política real de acogida de los refugiados y que dé respuesta a las necesidades de los ciudadanos de los países en guerra o en conflicto, así como las severas restricciones en inmigración y el férreo control de las fronteras exteriores de la UE mediante el sistema Frontex, empuja a miles de personas a dejar sus países desesperadamente con un riesgo muy alto de perder sus vidas en el camino, como vemos cada día, especialmente en los últimos meses. Es por ello que me gustaría compartir con vosotros la necesidad de tener una respuesta pública mediante una manifestación o una concentración en Barcelona, que llame a un cambio sustantivo de la política europea de derechos humanos respecto a los refugiados y a los solicitantes de asilo, y a un replanteamiento de las leyes de inmigración y de los controles de fronteras para prevenir la actual sangría humana. La idea es que, junto con las asociaciones, partidos y sindicatos que se adhieran a la iniciativa, debería haber un manifiesto, así como una convocatoria para un evento que debería tener lugar en Barcelona durante el mes de mayo.

Detalles de la reunión:Lunes, 20 de abril

$19 \mathrm{~h}$

Ciemen (Rocafort, 242) 
Este correo es un primer paso para la creación de SMM, que surgirá de manera natural a partir del intercambio de opiniones en esa reunión y en otras sucesivas. Entre los receptores de este email se encuentra E6, que asiste a esa primera reunión y a todas las demás. Ella será a la postre una persona muy comprometida y activa en SMM, con responsabilidades logísticas. Demostrando una capacidad de gestión de grupos y de personas y, siendo muy valorada por los demás miembros de la organización, la historia personal y familiar de la entrevistada está fuertemente marcada por la guerra civil española, ilustrando algunos vínculos personales con la migración y el asilo. "Tengo 23 años. Nací en Guatemala $y$ ahora vivo en Barcelona. Soy nieta de refugiados de Zaragoza (por parte de madre) y de Colombia (por parte de padre). Mi abuela fue una anarquista que vivió cuando la guerra civil española y supongo que esto es algo que te marca". Como muchos de los jóvenes activistas en el movimiento de solidaridad con las personas refugiadas (y en otros movimientos sociales actuales), el punto de inflexión en su socialización política tuvo lugar durante el 15M; esto es, la campaña de protesta de los Indignados que tomó las plazas durante meses a partir del 15 de mayo de 2011 (Tejerina y Perugorría 2017). "Cuando ocurrió el 15M, yo tenía solo 18 años. Lo viví como un acto de rebelión y con gran entusiasmo, porque estaba descubriéndolo todo. Estaba descubriendo palabras como feminismo, antirracismo, el movimiento anticapitalista... y las descubrí allí, mientras acampaba en las plazas" (Entrevista E6).

La campaña de movilización del 15M fue instrumental en la creación de nuevos partidos políticos como Podemos, y también fortaleció redes de activismo en una serie de cuestiones sociales, como la migración y el antirracismo (Portos 2019, 2021). El legado de la protesta en las calles incluyó la construcción de vínculos personales y de alianzas amplias entre la diversidad de personas que se habían conocido durante las ocupaciones, y permitió que los esfuerzos de movilización pudiesen emplearse en otros temas relacionados. La experiencia de E6 nos ofrece un buen ejemplo en este sentido: "Tras el surgimiento del 15M, el Ministro de Justicia decidió quitarnos el derecho al aborto y yo no podía aceptarlo. Así que el primer tema que me atrajo fue el feminismo y el derecho a nuestro cuerpo. En ese contexto, conocía a personas que trabajaban con víctimas del tráfico de personas. Al tratar con el feminismo migrante, empecé a tener conciencia de los movimientos por los derechos de los migrantes. Seguí en contacto con personas activas en movimientos de migrantes y hace medio año recibí una llamada para participar en una asamblea para tratar el hecho de que el fin de la operación Mare Nostrum había supuesto 900 muertos en 10 días." (Entrevista E6). Precisamente, estos eventos darían lugar- y nombre- a la plataforma SMM. ${ }^{5}$

En su manifiesto fundacional SMM reclamaba, entre otras medidas: cerrar FRONTEX ${ }^{6}$, un marco común para toda la UE sobre el asilo, terminar con las 2021).

${ }^{5}$ Véase la página web de SMM: https://stopmaremortum.org/ (consultada el 26 de julio de ${ }^{6}$ FRONTEX (Agencia Europea de la Guardia de Fronteras y Costas) es una agencia de la 
intervenciones extranjeras tanto en el terreno de la migración como las militares, un mayor control de los acuerdos comerciales (incluyendo el fin de la venta de armas a países donde tenían lugar violaciones masivas de derechos humanos) y el uso de fondos de cooperación al desarrollo y de ayuda humanitaria en este ámbito. A nivel organizativo, la plataforma consistía en una asamblea general, un comité ejecutivo y una serie de comisiones específicas formadas por los activistas, teniendo en cuenta que los miembros podían ser tanto individuos como organizaciones de la sociedad civil. Esta plataforma es un ejemplo poco frecuente de una organización que, buscando desafiar las políticas actuales regionales, nacionales y europeas de migración y asilo, consiguió convertirse en un nodo clave de la red catalana de solidaridad con las personas refugiadas y en un modelo para todo el Estado e incluso a escala internacional.

\section{MARCOS ESTRATÉGICOS, EL CARÁCTER TRANSVERSAL DE SMM Y EL COMPONENTE EMOCIONAL}

Esta convocatoria presenta elementos significativos de la futura estrategia y de los marcos de SMM, que serán ligeramente modificados y pulidos en posteriores comunicados de prensa. Por lo que respecta a la estructura organizacional, los objetivos iniciales no eran muy ambiciosos. Así, no hay nada en la convocatoria que explícitamente mencione la necesidad de crear una organización permanente. Los objetivos a corto plazo son dos: redactar un manifiesto y llevar a cabo un evento de protesta al cabo de unas semanas. En cuanto a la categoría de personas que merecen la ayuda de los activistas, hay un rechazo explícito a la distinción entre migrantes y personas refugiadas. Así, en varias ocasiones, los términos 'solicitantes de asilo y 'migrantes' se presentan conscientemente juntos. El mensaje es claro y directo: ambas categorías son merecedoras de los mismos derechos. Las sociedades europeas deberían ser capaces de acoger a ambos, tanto aquellos que escapan de la guerra, como a los que huyen de la precariedad económica. ${ }^{7}$

Las teorías de marcos enfatizan los procesos interpretativos culturales que median la atribución de significados a objetos, eventos y experiencias (Goffman 1974). Para reclutar a nuevos miembros y continuar motivando a los que están ya comprometidos, es necesario desarrollar marcos, entendidos como las visiones que guían y coordinan el comportamiento de actores individuales y colectivos (Snow y Benford 1988). Así pues, desde una perspectiva de marcos estratégicos, la convocatoria enfatiza la responsabilidad de la Unión Europea, un actor que se presenta como el principal blanco de la protesta. El marco diagnóstico, que tiene que ver con la identificación y las atribuciones del problema (Snow y

Unión Europea encargada de la gestión y el control fronterizo del área Schengen https://frontex. europa.eu/about-frontex/who-we-are/foreword/ (consultada el 26 de julio de 2021).

${ }^{7}$ El manifiesto de la convocatoria a la manifestación del 17 de junio de 2017 confirma esta elección consciente. Véase https://stopmaremortum.org/manifest-17j-obrimfronteres/. 
Benford 1988), también es claro: se culpa a la falta de una política genuinamente de asilo a escala europea, así como a la gestión de las fronteras europeas. En este sentido, diversos activistas también usan marcos anticapitalistas. "En vez de crear vías seguras hemos fortalecido las fronteras, hemos creado Frontex y seguimos aumentando su presupuesto. La crisis es solo la punta del iceberg. Los refugiados no son el problema, es el sistema el que hay que cambiar. Los refugiados representan una de las formas más visibles de violencia capitalista, que también crea guerras: la violencia de las fronteras" (Entrevista E3). En cuanto a la prognosis, a fin de solucionar este problema, se demandarán vías seguras, así como una profunda reorientación de las leyes migratorias y los controles de fronteras. Respecto a los marcos motivacionales, se menciona en varias ocasiones la interminable sucesión de muertes en el Mediterráneo en los últimos meses. La sensación de urgencia se destaca además por el hecho de que la reunión tendrá lugar al día siguiente (en menos de 24 horas). Un espacio de tiempo tan corto entre la convocatoria y la asamblea muestra la confianza que tenían los organizadores en el éxito del evento. Sin embargo, el nivel de asistencia superó las previsiones más optimistas.

A ello quizás contribuyó el momento en el que se envió la convocatoria. "Podría haber sido una semana antes o un mes después, pero ese era el momento adecuado; teníamos que hacer algo" (Entrevista E9). En abril de 2015 habían ocurrido varios naufragios en el Mediterráneo, que se saldaron con miles de muertos. ${ }^{8}$ La persona que redactó y promovió la convocatoria explica que esta no fue consecuencia de un evento concreto, sino de las continuas muertes en aquellos meses (Entrevista E9). Por lo que respecta a sus objetivos y a su relación con otros actores, no buscaban reemplazar, sino complementar los esfuerzos que las organizaciones de la sociedad civil estaban llevando a cabo en este ámbito. En este sentido, un activista involucrado en SMM, define su papel concreto en el conjunto de organizaciones trabajando en la solidaridad con las personas refugiadas:

"Cuando empezamos, ya había gente trabajando en las causas del problema. Esto es, la guerra. El movimiento pacifista aquí tiene una larga tradición y sus principales organizaciones han estado haciendo campañas contra el comercio de armas y en relación a los conflictos de Oriente Medio, por ejemplo. Por otro lado, también había ONGs conocidas que se dedicaban a acoger a los refugiados que ya habían llegado hasta aquí, como el Comité Catalán de Ayuda al Refugiado. Pero entre medio había un vacío. Nadie se ocupaba de la transición desde que estas personas dejan sus países de origen hasta que llegan a nuestra sociedad. Y ahí es donde vimos nuestro nicho; esto es, reclamar un pasaje seguro para los refugiados que están en camino. De esta manera, la organización tenía que convertirse en esencialmente política, porque tendría como objetivo conseguir un cambio en las políticas (a escala catalana, a escala española e incluso a escala europea), pero también en la percepción de los solicitantes de asilo entre la población catalana” (Entrevista E5).

\footnotetext{
${ }^{8}$ Véase, por ejemplo, Edwards y Dobbs (2015) y Malm et al. (2015).
} 
Ya en la primera reunión se obtuvieron algunos resultados concretos. ${ }^{9}$ En primer lugar, se decidió que se crearía una nueva plataforma social, centrada específicamente en las personas refugiadas en tránsito: SMM. Por otro lado, se redactó una nueva convocatoria para una manifestación que tendría lugar en pocas semanas. Y, finalmente, se acordó un lema potente: "Paremos el genocidio en el Mediterráneo". Ahora bien, el iniciador (E9), recuerda que no todos apoyaban la palabra 'genocidio'. La mayoría de participantes decidieron mantenerla, pese a que ello significase que algunas ONGs (por ejemplo, Amnistía Internacional) se iban a caer de la lista de firmantes del primer manifiesto.

El debate sobre la elección del lema arroja luz acerca de una de las principales características de la plataforma, destacada por la mayoría de entrevistados: su carácter heterogéneo y transversal. De acuerdo con E6, "desde la primera asamblea ha habido una pluralidad de gente muy diversa y hasta ahora hemos conseguido mantenerla. No conozco ningún otro espacio en el que anarquistas y cristianos trabajen conjuntamente. Como una de las más punkies de este grupo, tengo que admitir que al principio no estaba del todo segura de esto, porque he trabajado en temas de género y alli había personas con quienes me había enfrontado en manifestaciones pro-vida. Pero estábamos de acuerdo en el asunto de los refugiados. Este es uno de los aspectos de los que me siento más orgullosa en SMM" (Entrevista E6). Poco a poco, muchos migrantes se fueron implicando en las actividades de SMM, que se ha convertido en un ejemplo para construir alianzas entre inmigrantes y otros actores de la sociedad civil (García Agustín y Jørgensen 2016: 226-230). De hecho, SMM pasó a jugar un papel como plataforma paraguas que coordinaba la acción de diferentes grupos y organizaciones que se ocupan del tema de las personas refugiadas. En palabras de otro activista, "en el contexto de SMM, se hicieron intentos para conseguir convocatorias unitarias. Antes de que naciésemos, ONGs como CEAR, AAPS (Asociación de Apoyo al Pueblo Sirio) o SOS Racisme convocaban una manifestación y luego los demás se sumaban. Nuestro reto era hacer las cosas de una manera distinta" (Entrevista E2).

Sin embargo, dar cobijo a esta heterogeneidad y convertirse en la piedra angular del movimiento de solidaridad con las personas refugiadas no eran los principales objetivos de SMM en un principio. Más bien fueron el resultado de su evolución estratégica, a medida que la plataforma fue adaptando sus aspiraciones de desempeñar un papel más relevante en la política catalana. "El tema de la coordinación no lo planeamos en un inicio. A veces hay personas que quieren hacer algo, se cogen a un paraguas mayor y en este sentido también hemos acogido otras iniciativas o las hemos compartido" (Entrevista E5). Como destacaba otro activista, "parecía imposible hace un año conseguir el nivel de reconocimiento mediático que tenemos ahora. Queremos cambiar los discursos mediáticos, pero también las políticas, al menos en nuestro país. Estamos convencidos de que si hay un cambio significativo en Cataluña, puede ser un modelo para

${ }^{9}$ Hay que destacar que a esta primera reunión ya participaron una quincena de personas que venían de entornos de la inmigración (Entrevista E6). 
otros lugares; esto es, una medida de lo que puede ser posible. Nuestro éxito a la hora de cerrar el CIE puede entenderse desde esta perspectiva" (Entrevista E9). De algún modo, los eventos que tuvieron lugar tras la primera reunión de SMM sobrepasaron las previsiones y expectativas de los activistas. "Era inconcebible que pudiese alcanzar el nivel de movilización que ha conseguido" (Entrevista E7). Otro activista confirma esta idea: "todo lo que vino después era mayor que nuestra capacidad organizativa. Lo que ocurría era tan serio y las respuestas europea y española (por parte de los decisores políticos) era tan decepcionantes que estaba claro que la opinión pública demandaba un cambio en las políticas sobre inmigración y asilo" (Entrevista E2).

El estudio de las emociones ha desempeñado un papel secundario en las investigaciones sobre movimientos sociales entre los años 1970, y al menos hasta inicios de los 2000 (Goodwin, Jasper y Polletta 2004). No obstante, la dimensión emocional es fundamental para entender la capacidad de SMM para abarcar distintas sensibilidades. A escala individual, hemos hallado que la empatía era la principal razón, el principal valor que había tras la decisión de participar en este movimiento social. A un nivel básico fenomenológico, la empatía denota una respuesta afectiva al estado emocional de otro ser humano, ya sea directamente percibido, imaginado o inferido (Lamm y Silani 2015: 64). En muchos casos, la empatía puede resultar en preocupación y compasión, y esto, a su vez, en un activismo social (Hess, Houde y Fischer 2014).

Tal y como ilustra el caso de E6, la empatía está habitualmente ligada a la historia personal y familiar del activista. Así, la mayor parte de personas entrevistadas tienen una historia personal ligada a uno o a varios de los siguientes eventos o movimientos: el 15M (2011), la Guerra Civil (1936-1939), la PAH (desde 2009), guerras y desplazamientos forzados en la ex-Yugoslavia, Sáhara, Palestina, América Central o Colombia. La historia del iniciador del llamamiento que daría pie a SMM, es particularmente relevante. "He sido responsable de un programa de televisión sobre asuntos migratorios; gracias a ello forjé los contactos necesarios para movilizar a un grupo de personas y comenzar un proceso de reflexión colectiva. Tanto a nivel personal como profesional, la migración es un tema recurrente en mi vida. Cuando comencé a trabajar en asuntos sociales en Cataluña había una tasa de inmigrantes del 4\%, pero creció de manera constante hasta el $17 \%$ en 2008. Pero entonces, cuando llegó la crisis económica, estas cifras comenzaron a declinar" (Entrevista E9). El mismo entrevistado continúa:

“'Abrid las fronteras, queremos acoger' fue el eslogan de una de las manifestaciones de 2016. La ciudadanía catalana abrió sus puertas, ofreciendo sus casas para dar cobijo a una familia siria, como alternativa a la falta de políticas de asilo por parte de los Estados. En el caso de Barcelona, uno de los principales precedentes tuvo lugar en 1994. En un exitoso ejemplo de colaboración ciudadana, unos 300-400 refugiados vinieron aquí durante la guerra de Bosnia. Por aquel entonces, la Unión Europea no estaba tan consolidada como ahora, y los Estados no se coordinaban en muchos temas. Alguna gente se acordaba de lo que pasó entonces y otros recordaban su propia historia durante la Guerra Civil. En mi caso, mi familia se quedó de 
este lado [de la frontera] en campos de concentración simplemente porque no consiguieron huir. Si hubiesen podido, habrían sido refugiados. En mi casa yo siempre he escuchado 'tú podrías haber nacido en otro lugar' porque mis padres emigraron dos veces en los años 1960. Soy catalán por pura casualidad" (Entrevista E9).

Antes del estallido de la Gran Recesión, distintas organizaciones habían luchado por las condiciones de la vivienda digna y contra las ejecuciones hipotecarias (particularmente, V de Vivienda y la PAH) — véase Aguilar Fernández y Fernández Gibaja (2010). Gran parte de las personas víctimas de desahucios han sido inmigrantes internacionales. Múltiples espacios y colectivos, incluyendo por ejemplo a Banc d'Aliments, se organizaron para ayudar a los colectivos más desfavorecidos, proporcionando comida, techo, etc. De acuerdo con activistas de la propia SMM, "nuestra plataforma también se apoya sobre este tejido social" (Entrevista E2).

De hecho, tanto en Lampedusa como en Idomeni, la presencia de activistas de origen catalán ha sido notable. Son muchas las organizaciones catalanas que se han dedicado a la cooperación internacional, especialmente desde los años 1980 (Muñoz Márquez 2011). Estas redes de ayuda al desarrollo, muchas de ellas involucradas en el movimiento por la justicia global a finales del S.XX, son clave para entender tanto el nacimiento como la capacidad de movilización de las organizaciones de ayuda a personas refugiadas. Como contrapartida, esta exposición a redes internacionales de cooperación contribuye a la concienciación y despierta sentimientos de empatía, entre otras emociones. En palabras de una activista, "mientras que en otros contextos podría ser caldo de cultivo para el fascismo, aquí es una tierra fértil para la solidaridad" (Entrevista E8). Por emociones nos referimos a la expresión de afecto- respuestas corporales inconscientes a estímulos- a través de convenciones sociales o culturales (Gould 2009: 18-22). Estas emociones, en cuanto esquemas interpretativos, también se forjan durante la acción. Con respecto a un evento de protesta en favor de las personas refugiadas, una activista manifiesta sentirse del siguiente modo:

"Tengo emociones encontradas. Estoy enfadada, pero duele. Ellos (los refugiados) me recuerdan a mi abuela escapando de la guerra. Porque he estado en los campos (de refugiados) y conozco los nombres y apellidos de muchos refugiados, pienso en ellos. Veo que la gente los insulta en las redes sociales, pidiendo gasearlos... cuando protestamos, cuando nos manifestamos, pienso en los refugiados, en cómo se sentirán cuando les muestre las fotos de esta protesta hoy, y en toda la fuerza que esto les va a dar" (Entrevista E6).

Hay otra palabra que resuena en nuestras entrevistas: impotencia. Aunque a priori éste no sea un sentimiento positivo de cara a impulsar a la acción, en el seno de SMM en varias ocasiones se ha transformado en un sentimiento que favorece la movilización. Este trabajo de gestión emocional se desarrolla para presentar el "yo" que se espera ante una injusticia, de modo que encaje con las expectativas sociales generadas (Hochschild 1983). De acuerdo con un activista: 
"Lo que ha sucedido en el Mediterráneo, y esto incluye la Frontera Sur (aunque puede que lo hayamos olvidado, todavía está ahí para recordarnos que las políticas europeas sobre asilo e inmigración han sido iguales desde hace mucho), la cantidad y el flujo de llegadas han sido más evidentes desde la guerra en Siria. Ha habido muchos muertos. ¿Qué podemos hacer como ciudadanos? Podemos presionar a los políticos para subrayar los fallos en las politicas y comenzar a hablar sobre alternativas" (Entrevista E3).

Con una mirada retrospectiva, aunque la evaluación generalizada por parte de los entrevistados sobre el trabajo realizado por SMM sea optimista, gran parte de los activistas reconoce que no todos los objetivos se han logrado todavía. "Un año después de su creación, SMM se ha convertido en un elemento relevante de presión, pero no lo suficientemente efectiva. Quedan aún muchas cosas por conseguir. Somos persistentes, y la persistencia es el primer caso para cambiar las políticas. Nuestras tres principales áreas de trabajo son la movilización, el apoyo y la concienciación" (Entrevista E5). Y precisamente, en la siguiente sección arrojaremos luz sobre la evolución estratégica de SMM, centrándonos en una serie de características organizacionales que han incidido en su capacidad de movilización.

\section{LA EVOLUCIÓN ESTRATÉGICA DE SMM: EVENTOS TRANSFORMATIVOS, REPERTORIOS DE ACCIÓN E INTERACCIONES CON ACTORES CLAVE}

Desde la literatura de movimientos sociales, varios autores han argumentado que ciertos eventos pueden poseer un carácter transformativo; es decir, son "puntos de inflexión cruciales" (Hess y Martin 2006: 249; McAdam y Sewell 2001). Esos eventos transformativos pueden tener impactos en los propios movimientos que los llevan a cabo en distintas dimensiones, incluyendo la cognitiva, la afectiva y la relacional (Della Porta 2008; Portos 2019; Portos y Carvalho 2019). Estos cambios, argumentamos en este artículo, pueden moldear la capacidad organizativa a nivel local y aumentar o hacer decrecer de forma dramática los niveles de movilización (véase Hess y Martin 2006).

En concreto, a medida que la crisis de personas refugiadas se fue desarrollando, podemos identificar dos eventos transformativos clave que han impactado en la capacidad de movilización. Por una parte, la foto del niño sirio ahogado Alan Kurdi, cuyo cuerpo fue encontrado yaciendo boca abajo en una playa cerca del resort turco de Bodrum, conmovió a gente con un perfil distinto al habitual del activista y contribuyó a aumentar el interés sobre la situación de los refugiados sirios, y también el apoyo hacia (y la movilización en) el movimiento en solidaridad con las propias personas refugiadas. Es más, este evento incrementó la actividad en las redes organizacionales, dando a pie al nacimiento de distintos colectivos. El siguiente extracto de la entrevista con un miembro de la plataforma local de apoyo a personas refugiadas en el distrito Sagrada Familia ilustra cómo esta organización nació precisamente para ocuparse de este tema: "Mucha 
gente del barrio vio en las noticias lo que estaba pasando en el Mediterráneo, queríamos ayudar y no confiábamos en el Gobierno. Entonces empezamos a organizarnos y a preguntarnos: ¿¿qué podemos hacer?" (Entrevista E4). Otra entrevistada confirma esta idea: "en mayo de 2015 no había más de una treintena de personas en nuestras manifestaciones. En septiembre de 2015 éramos más de quinientas" (Entrevista E6). Sin embargo, algunos entrevistados añaden una nota de precaución: "la cuestión es cuánta de esta gente permanecerá con nosotros a largo plazo, una vez que la atención mediática disminuya" (Entrevista E9).

El segundo punto de inflexión significativo en la trayectoria de SMM concierne la firma del acuerdo migratorio entre Turquía y la Unión Europea en marzo de 2016 (Kingsley y Rankin 2016). "Paradójicamente, el acuerdo ayudó a nuestro movimiento. Justo antes, estábamos poniendo demasiado énfasis en algunas diferencias internas que teníamos; este acuerdo fortaleció la cohesión del grupo. Además, nuestro acto de protesta denunciando el pacto tuvo mucha repercusión en los medios, y el hecho de que funcionó tan bien hizo que mucha gente se interesase en colaborar con nosotros" (Entrevista E6). Aquella manifestación de SMM, que congregó a más de 15.000 personas, conllevó un gran poder simbólico: por primera vez, representantes políticos encabezaron la marcha, reflejo de cómo SMM se había convertido ya en un actor clave y referente de la sociedad civil. En la primavera de 2016, un gran número de eventos en solidaridad con las personas refugiadas tenía lugar prácticamente a diario en Barcelona; pero la mayoría de estos eventos fueron pequeños y escasamente coordinados entre sí- en buena medida, debido a la naturaleza policéfala y descentralizada de las redes de activistas en la ciudad condal (véase, por ejemplo, Yates 2015). No obstante, en la evolución del movimiento se observa una clara tendencia hacia la coordinación y el desarrollo de acciones unitarias bajo el paraguas de SMM.

De hecho, los propios actos de protesta se encuentran en el corazón de SMM. A lo largo de 2015 y 2016 la plataforma promovió múltiples eventos en distintos puntos de referencia, incluyendo concentraciones multitudinarias ante la sede de la Comisión Europea en Barcelona (La Vanguardia 2016). Por ejemplo, activistas de SMM acamparon y colgaron un cartel gigante en la fachada del edificio de la Pedrera cuando la alta representante de la Unión para Asuntos Exteriores y Política de Seguridad, Federica Mogherini, entraba en el Parlamento Europeo (Camps 2016). También llevaron a cabo una impactante performance rociando de pintura roja a activistas que sostenían un cartel con la palabra "vergüenza" ante el pacto entre la Unión Europea y Turquía. En febrero de 2017, decenas de miles marcharon en el centro de Barcelona exigiendo al gobierno la acogida de más personas refugiadas; este evento se convertiría, hasta aquel entonces, en la manifestación en favor de los derechos de las personas refugiadas más concurrida de la historia en toda Europa (Safont 2017; The Guardian 2017). Estas acciones ayudaron a SMM a adquirir un impacto mediático notable, 
incluso con reportajes sobre la organización en medios internacionales de primer nivel, que a su vez ilustran la expansión del movimiento. ${ }^{10}$

Una de las razones que explican la capacidad de SMM para movilizar a unas bases tan transversales radica en sus repertorios de acción, con un diseño muy cuidadoso y que emplean tácticas mixtas. De acuerdo con un entrevistado, "al principio el único tipo de protesta que podíamos desarrollar era una cacerolada armados de ollas y sartenes delante de la Delegación de la Unión Europea, y quizás algunas concentraciones aisladas. Cualquier manifestación en aquel momento hubiese sido un fracaso. Incluso nuestros simpatizantes más acérrimos se habrían quedado decepcionados. Debemos ser muy rigurosos cuando hablamos de organizar una manifestación; necesitamos saber que va a tener éxito" (Entrevista E2). La preocupación por el éxito y el control de la táctica denota una cierta experiencia política, así como ocasionales desacuerdos y tensiones en el seno del grupo sobre las tácticas a utilizar (E2; E6). Los activistas de SMM deliberadamente intentaron ir más allá de la protesta y formas de acción disruptivas. "Queríamos que nuestras acciones fuesen más que meras concentraciones. Para ello, necesitábamos algo diferente que atrajese a gente nueva, como la participación de un artista (gráfico, escénico, musical). Cuanto más visual, mejor. De este modo, ofrecimos acciones colectivas pacíficas, sin confrontación, donde todo el mundo se podía sentir cómodo e involucrarse" (Entrevista E6).

Junto con las acciones de protesta y performances públicas, otra de las acciones más frecuentes de la plataforma ha consistido en despertar la concienciación sobre el asunto de las personas refugiadas. De hecho, "además de las pequeñas reuniones que se hacían cada lunes, nuestras acciones más comunes han sido charlas públicas para concienciar a la ciudadanía". En palabras de esta activista, "tuvimos que crear asambleas públicas porque más y más gente venía a nuestras reuniones internas. Empezamos a tener que decir 'no' a las invitaciones a dar charlas porque no dábamos abasto. Hemos llegado a dar siete charlas en un solo día. Recibimos muchas invitaciones de pueblos que están lejos de Barcelona. Hemos recorrido todo el territorio catalán, que es complicado desde el punto de vista logístico. Pero ésta es la gente a la que es importante llegar" (Entrevista E6). Otro frente de acción importante y complementario para SMM ha sido la persuasión política: "Presionamos desde las calles, y bien el mismo día o el día siguiente, presionamos a las instituciones. Es una dirección bidireccional. Por ejemplo, sabemos que pasar una moción en el Parlamento es mucho más fácil si ha habido una manifestación el fin de semana anterior" (Entrevista E5). Buena parte de estas actividades de petición fueron online, o requirieron la difusión a través de plataformas digitales. En resumen, el repertorio de acción de SMM ha estado en permanente evaluación, combinando acciones locales con actividades de concienciación, semi-institucionales y eventos de protesta cuidadosamente diseñados y ejecutados para obtener la máxima repercusión pública posible.

10 Véase BBC (2017). 
$\mathrm{Al}$ margen de la importancia de eventos transformativos y de los repertorios de acción, un tercer aspecto que ha incidido en la configuración estratégica de SMM a lo largo del tiempo tiene que ver con la interacción organizacional con otros actores relevantes. Si bien SMM se convirtió en el principal actor dentro de la red de solidaridad con personas refugiadas en Barcelona, no es la única organización activa en este ámbito- de hecho, más de un centenar de organizaciones se manifestaron el 19 de Junio de 2016 en Barcelona durante el Día Mundial del Refugiado (Ara 2016). Entre estos colectivos destacan organizaciones establecidas en las áreas de migración, antirracismo, pacifismo o antimilitarismo, como la Cruz Roja, CEAR, o SOS Racisme. De hecho, existe mucho solapamiento entre los miembros de organizaciones como SOS Racisme y SMM. Otras entidades, en cambio, son de naturaleza más reciente. Por ejemplo, Fotomovimiento es una organización centrada en la documentación audiovisual de movilizaciones sociales- en estos años fundamentalmente sobre el tema de las personas refugiadas-, y que tiene su génesis en las asambleas del 15M. Así, un entrevistado recorrió la ruta balcánica con su cámara de fotos; al regresar a Barcelona, organizó una exposición fotográfica para mostrar "lo que de verdad está pasando allí, algo que difícilmente encontrarás en los medios de comunicación convencionales" (Entrevista E3). Otra de las organizaciones de reciente creación, y que ha adquirido gran notoriedad pública, es ProActiva Open Arms. ${ }^{11}$ Nacida como una ramificación de una empresa de socorristas profesionales, esta ONG ha rescatado a miles de inmigrantes de las aguas del Mediterráneo..$^{12}$ En contraste con otras zonas de España, las organizaciones religiosas han jugado un rol marginal en Barcelona (Alcalde y Portos 2018). De acuerdo con un activista de SMM, "aunque algunas tienen un punto rebelde, el enfoque de estas organizaciones aquí [en Barcelona] es asistencial. Han firmado manifiestos, etc., pero hay un elemento siempre de salvar a la gente, de darles asistencia, en lugar de la confrontación política que nos caracteriza a nosotros. Nosotros necesitamos la confrontación política, porque queremos que las políticas cambien" (Entrevista E7).

En general, los activistas entrevistados tienden a definir la relación de SMM con el Ayuntamiento como "cordial", pero indican que los canales de comunicación son mejorables. Ya hemos enfatizado la heterogeneidad en el seno de la plataforma, que incluye también a miembros de distintos partidos políticos en el espectro ideológico de la izquierda. Ahora bien, los activistas más próximos a la coalición que apoya a Ada Colau, Barcelona en Comú, tienden a no ser los más activos en SMM, a pesar de su posición favorable y comprometida con la causa de las personas refugiadas. ${ }^{13} \mathrm{El}$ contexto particular de Barcelona refleja las ventajas e inconvenientes de contar con un potencial aliado en el poder a nivel municipal. En líneas generales, sabemos que la presencia de aliados institucionales tiende a aumentar los recursos de que disponen los movimientos,

11 Véase https://www.proactivaopenarms.org/.

12 El documental Astral, que cuenta la historia del barco usado por la asociación, se estrenó en docenas de cines en España y en un canal de televisión nacional (La Sexta 2016).

13 Véase Colau (2015). 
presentando oportunidades para avanzar su agenda, ensanchar su base de apoyo e incidir en el proceso de toma de decisiones y diseño de las políticas públicas (Bazurli 2019). Gobiernos municipales y movimientos sociales ideológicamente congruentes poseen incentivos para construir alianzas que permiten a los actores obtener réditos políticos y moldear políticas en un contexto poco receptivo y hostil a las proclamas del movimiento- y Barcelona no ha sido una excepción en este sentido (García Agustín y Jørgensen 2018). No obstante, y como efecto colateral, han surgido disputas internas sobre las distintas rutas estratégicas que SMM debe adoptar con las instituciones, revelando las tensiones entre lógicas más colaborativas o beligerantes para con las instituciones en general, y particularmente hacia el consistorio barcelonés. En este sentido, una parte de los activistas tiene una posición crítica con la alcaldesa, al entender que pretende obtener réditos políticos a través de un asunto acuciante, pero sin contribuir a transformar sustancialmente la situación. En palabras de una activista:

"Este es un movimiento que nace desde abajo y sigue siendo así. No puede estar controlado por el Ayuntamiento de Barcelona o por el Govern catalán. En alguna ocasión, gente próxima al ayuntamiento ha intentado ocupar nuestro lugar. Existe una orientación política muy específica del ayuntamiento, que claramente intenta liderar la lucha por los derechos de los refugiados. Yo estoy convencida que de verdad creen en esta causa, pero se traduce en una ventaja política para ellos. Desde el punto de vista electoral, este es un tema atractivo en Barcelona” (Entrevista E2).

En cuanto a los oponentes del movimiento, "solamente los turistas a veces critican nuestras protestas" (Entrevista E6). Esta situación es un rara avis en el contexto de la Unión Europea. La crisis de refugiados tuvo lugar en un momento en que varios países estaban cuestionando sus políticas migratorias, con la proliferación de movimientos xenófobos y racistas en países como Francia, Italia, Grecia, Holanda y Alemania. En Barcelona, la presencia de grupos ultra que tiene a las personas refugiadas en el punto de mira era marginal - al menos hasta 2017.

Si bien los activistas catalanes en temas migratorios reciben ataques de forma habitual a través de las redes sociales, en el período 2015-2017 no hubo en Barcelona un solo evento de protesta organizado contra la acogida de personas refugiadas. Varios factores podrían dar cuenta de ello. Una primera explicación podría radicar, en primer lugar, en que la pequeña cantidad de personas refugiadas en la ciudad no genera problemas de convivencia- sin embargo, en otros países donde el número de refugiados es prácticamente insignificante, como en Polonia, el nivel de oposición y contestación a los mismos ha sido sin embargo muy significante. En segundo lugar, la organización y movilización de la sociedad civil en solidaridad con los personas refugiadas puede haber contribuido al fracaso de los marcos discursivos contrarios a la acogida. A ello puede haber ayudado, por una parte, el hecho de que exista un fuerte movimiento de base antirracista y antifascista muy arraigando históricamente en Barcelona, y por otra el que varios grupos de inmigrantes hayan estado sobrerrepresenta- 
dos y muy activos en iniciativas sociales y organizaciones que gozan de gran legitimidad popular, como la PAH. En tercer lugar, desde el punto de vista de la movilización social, en este período se sucedieron movilizaciones contra la austeridad y la autodeterminación e independencia, que han prefigurado marcos democrático-emancipatorios e inclusivos (Della Porta et al. 2017), que son difícilmente compatibles con concepciones insolidarias sobre el asunto de las personas refugiadas. Particularmente, la campaña de movilización de los indignados puede haber facilitado la canalización del descontento ciudadano de un modo más tolerante e inclusivo con respecto a otros países. En cuarto lugar, la estructura de clivajes española en general, y la visibilidad del conflicto centroperiferia, en particular, son aspectos que han sido negativos para las expectativas electorales de los partidos de derecha populista radical — también castigados por el propio sistema electoral (Alonso y Rovira-Kaltwasser 2015). Asimismo, el sistema de competición partidaria y las estrategias electorales del Partido Popular favorecían la movilización electoral tanto a sectores moderados como muy escorados a la derecha. Durante años, el PP se convirtió en un partido dominante en el espectro ideológico de la derecha - al menos hasta la irrupción del partido de centro-derecha liberal Ciudadanos y la fuerza antinmigración y de extrema derecha VOX, que ha venido a cuestionar el excepcionalismo español en cuanto a la inexistencia de partidos populistas con representación parlamentaria.

\section{CONCLUSIÓN}

A diferencia de otros países mediterráneos como Grecia o Italia, España no es un país que haya experimentado un gran incremento de demandantes de asilo a la luz de los flujos de personas desplazadas a la fuerza, procedentes de países africanos y de Medio Oriente. A pesar de la cerrazón institucional de un gobierno estatal no particularmente sensible al drama, un sólido movimiento de base en solidaridad con las personas refugiadas ha emergido en el país en los últimos años. Examinando el contexto local de Barcelona, este artículo ofrece dos contribuciones a la literatura existente.

En primer lugar, contribuye a cubrir un vacío en el estudio de los movimientos sociales. Centrándose en el meso-nivel de análisis, explora los factores del éxito de la organización SMM. Esta plataforma se convirtió en un nódulo central en la red del movimiento en solidaridad con las personas refugiadas de Barcelona, mostrando una importante capacidad de movilización social. Como hemos visto, muchos activistas manifiestan un historial de activismo previo, así como lazos personales con el sector de la inmigración y las personas refugiadas, pero la configuración del movimiento dista de ser estable a lo largo del tiempo. Aunque algunos eventos tuvieron una capacidad transformativa clave en la trayectoria de la organización, SMM consiguió llevar a cabo una mezcla de tácticas confrontacionales y semi-institucionalizadas, de concienciación y generalmente formas de acción menos disruptivas para conseguir presencia mediática, movilizar a la sociedad civil e influir en el diseño de las políticas públicas. Asimismo, 
la dimensión emocional ha jugado un papel importante en las razones formuladas por los activistas para dar cuenta de su implicación.

Por otra parte, hemos enfatizado la importancia de las relaciones e interacciones de SMM con otros actores sociales, así como con las instituciones, en el área de la solidaridad con las personas refugiadas. A pesar de la fuerza y legitimidad social que ha adquirido SMM, no se ha convertido en el actor hegemónico en su sector, sino que cohabita en una compleja red de actores, algunos con una larga trayectoria de movilización, otros nacidos a la luz de la crisis de 2015 en el Mediterráneo. Desde una perspectiva comparada, una característica distintiva de la red de solidaridad con las personas refugiadas en Barcelona radica en el hecho de que no existen actores organizados contra este movimiento social. Asimismo, la presencia de una alcaldesa partidaria del movimiento y su causa abre oportunidades para la colaboración y la creación de sinergias, pero al mismo tiempo crea tensiones acerca de qué enfoques estratégicos se deben adoptar. Este tema abre una ventana que requiere de una investigación empírica más profunda: ¿en qué medida la presencia de aliados institucionales a nivel local - y una estructura de oportunidades políticas cambiantes en general- contribuye al éxito organizacional? La evidencia preliminar sobre el contexto español procedente de otros casos distintos al de Barcelona sugiere que, mientras las candidaturas de unidad popular apoyadas por Podemos son más permeables y proclives a escuchar, apoyar e incorporar en su agenda las demandas del movimiento, éstas no se trasladan en políticas concretas ni necesariamente determinan la fortaleza ni la capacidad de movilización de las redes de activistas en pro de las personas refugiadas. Asimismo, es probable que exista cierto grado de asociación endógena entre algunos elementos considerados en este estudio: marcos y movilización, repertorios y estrategias tienden a retroalimentarse, por lo que futuros estudios podrán tratar de profundizar en las direcciones causales.

En qué medida los resultados de un caso de estudio son extrapolables a otras organizaciones- que se han desarrollado en ámbitos, contextos y circunstancias muy diferentes - continúa siendo una pregunta que exige de ulteriores análisis empíricos. Una línea interesante de investigación consistiría en ver qué consecuencias entraña la participación en una organización en la vida política del individuo, y cómo este compromiso se traslada - o no- en cambios sociobiográficos y la disposición a actuar, pensar y percibir de un modo acorde- $\mathrm{o}$ inconsistente- con la socialización previa (Fillieule y Neveu 2018).

Con el objetivo de arrojar luz sobre la evolución y la trayectoria organizacional de SMM, este artículo ha abogado por adoptar una perspectiva diacrónica para estudiar las dinámicas de movilización a nivel organizacional. Iniciada como una red activista a pequeña escala, esta organización se ha convertido en una plataforma paraguas que aglutina el grueso del movimiento de solidaridad con las personas refugiadas en Barcelona, mostrando una habilidad única para adaptar sus repertorios, estrategias y marcos ante un contexto rápidamente cambiante. Como contrapartida, esta capacidad para modular las características organizacionales ha permitido a SMM aumentar su capacidad de movilización y establecer una amplia y transversal base social. 


\section{BIBLIOGRAFÍA}

AGUILAR FERNÁNDEZ, S. y A. FERNÁNDEZ GIBAJA. (2010): “El movimiento por la vivienda digna en España o el porqué del fracaso de una protesta con amplia base social", Revista Internacional de Sociología, 68(3), pp. 679-704.

ALCALDE, J. (2016): "Por qué la crisis de los refugiados no es una crisis de los refugiados", en Por la paz / Peace in Progress, 29, noviembre 2016, http://www.icip-perlapau.cat/numero29/articles centrals/article central 2/

ALCALDE, J. y M. PORTOS. (2018): "Refugee solidarity in a multilevel political opportunity structure. The case of Spain”, en Solidarity Mobilisations in the 'Refugee Crisis': Contentious Moves, Londres, Palgrave Macmillan.

ANDRETTA, M., G. PIAZZA y A. SUBIRATS. (2015): "Urban dynamics and social movements", en The Oxford Handbook of Social Movements, Oxford, Oxford University Press.

ARA. (2016): "Un centenar d'entitats convoquen una manifestació a Barcelona en solidaritat amb els refugiats", 13 junio 2016, http://www.ara.cat/internacional/dentitatsmanifestacio-Barcelona-solidaritat-refugiats 0 1594640673.html.

ARCARONS, A. (2016): "Case Study Spain”, en From Refugees to Workers. Mapping Labour-Market Integration Support Measures for Asylum Seekers and Refugees in EU Member States, Vol. II: Literature Review and Country Case Studies, Bartelsmann Stiftung, pp. 107-122, https://www.bertelsmann-stiftung.de/de/publikationen/ publikation/did/from-refugees-to-workers-mapping-labour-market-integration-support-measures-for-asylum-seekers-and-3/.

ATAÇ I., K. RYGIEL y M. STIERL. (2016): "The contentious politics of refugee and migrant protest and solidarity movements: remaking citizenship from the margins", Citizenship Studies, 20(5), pp. 527-544.

BASTIA, T. (2011): "Should I stay or should I go? Return migration in times of crisis", Journal of International Development, 23, pp. 583-95.

BAZURLI, R. (2019): "Local Governments and Social Movements in the 'Refugee Crisis': Milan and Barcelona as 'Cities of Welcome'", South European Society and Politics, 24(3), pp. 343-370.

BBC. (2017): "Barcelona protest to support refugees draws thousands", 28 febrero 2017, https://www.bbc.com/news/world-europe-39017265.

BLEE, K. M. y V. TAYLOR. (2002): "Semi-Structured Interviewing in Social Research", en Methods of Social Movement Research, Minneapolis, University of Minnesota Press, pp. 92-117.

CAMPS, C. (2016): "Acampan ante la representación europea en Barcelona por los derechos de los refugiados", en El Nacional, 15 mayo 2016, https://www.elnacional. cat/es/sociedad/acamada-union-europea-barcelona-refugiados-pedrera-passeo-gracia 103185 102.html.

CEAR. (2015): Diez datos dolorosos sobre la realidad de los refugiados, 30 octubre 2015, https://www.cear.es/diez-datos-dolorosos-sobre-la-realidad-de-los-refugiados/.

COLAU, A. (2015): "We, the cities of Europe", Blog de la alcaldesa de Barcelona, 13 septiembre 2015, http://ajuntament.barcelona.cat/alcaldessa/en/blog/we-cities-europe.

COMISIÓN EUROPEA. (2015): “Gestión de la crisis de los refugiados: medidas operativas, presupuestarias y jurídicas inmediatas en el marco de la Agenda Europea de Migración", Press Release, 23 Septiembre 2015, http://europa.eu/rapid/press-release IP-15-5700 es.htm. 
DELLA PORTA, D. (2008): "Eventful Protest, Global Conflicts", Distinktion: Journal of Social Theory, 9(2), pp. 27-56.

DELLA PORTA, D. (ed.). (2018): Solidarity Mobilizations in the 'Refugee Crisis'. Contentious Moves, Londres, Palgrave Macmillan.

DELLA PORTA, D., F. O'CONNOR, M. PORTOS y A. SUBIRATS. (2017): Social movements and referendums from below: Direct democracy in the neoliberal crisis, Bristol, Policy Press/ University of Bristol.

EDWARDS, A. y L. R. DOBBS. (2015): "Latest deaths on Mediterranean highlight urgent need for increased rescue capacity”, UNHCR, 15 abril 2015, http://www.unhcr. org/news/latest/2015/4/552e603f9/latest-deaths-mediterranean-highlight-urgent-need-increased-rescue-capacity.html.

FIGUEREDO, E. (2015): "El CIE de la Zona Franca cierra sus puertas", en La Vanguardia, 23 Octubre 2015, http://www.lavanguardia.com/local/barcelona/20151023/54438318647/cie-barcelona-cierra.html.

FILLIEULE; O. y NEVEU, E. (Eds.) (2018): Activists Forever? Long-Term Impacts of Political Activism, Cambridge, Cambridge University Press.

GALARRAGA, N. (2017): "España, pocas peticiones de asilo y muy pocos refugiados", en El País, 1 marzo, https://elpais.com/internacional/2017/03/01/actualidad/1488370575_958936.html, consultado el 22 de junio 2021.

GARCÍA AGUSTÍN, Ó. y M. B. JØRGENSEN. (2016): “Against pessimism: a time and space for solidarity", en Solidarity without borders. Gramscian perspectives on migration and civil society alliances, London, Pluto Press.

GARCÍA AGUSTÍN, Ó. y M. B. JØRGENSEN. (2018): Solidarity and the 'Refugee Crisis' in Europe, London, Palgrave Pivot.

GOFFMAN, E. (1974): Frame Analysis: An Essay on the Organization of Experience, New York, Harper Colophon Books.

GOODWIN, J., J. M. JASPER y F. POLLETTA. (2004): "Emotional Dimensions of Social Movements", en The Blackwell Companion to Social Movements, Oxford, Blackwell Pub.

GOULD, D. B. (2009): Moving politics: Emotion and ACT UP's fight against AIDS, Chicago, Chicago University Press.

HELLGREN, Z. e I. SERRANO. (2017): "Transnationalism and financial crisis: the hampered migration projects of female domestic workers in Spain", Social Sciences, $6(1) 8$.

HESS, D. y B. MARTIN. (2006): "Repression, backfire, and the theory of transformative events. Mobilization", Mobilization, 11(1), pp. 249-267.

HESS, U., S. HOUDE y A. FISCHER. (2014): "Do we mimic what we see or what we know?", en Collective emotions, Oxford, Oxford University Press, pp. 94-107.

HOCHSCHILD, A. (1983): The Managed Heart: Commercialisation of Human Feeling, Berkeley, University of California Press.

KEMP, W. (2016): "Learning from the Canaries: Lessons from the "Cayucos" Crisis", en International Peace Institute, 31 mayo 2016. https://www.ipinst.org/2016/05/lessons-from-the-cayucos-crisis

KINGSLEY, P. y J. RANKIN. (2016): "EU-Turkey refugee deal - Q\&A", en The Guardian, 8 marzo 2016 https://www.theguardian.com/world/2016/mar/08/eu-turkey-refugee-deal-qa

LAMM, C. y G. SILANI. (2014): "The neural underpinnings of empathy and their relevance for collective emotions", en Collective Emotions, Oxford, Oxford University Press. 
LA SEXTA. (2016): ¿En qué cines podrás ver el estreno de 'Astral', la primera película documental de Jordi Évole en Salvados?, http://www.lasexta.com/programas/salvados/programa/que-cines-podras-ver-estreno-astral-primera-pelicula-documentaljordi-evole-salvados 2016092157e2cd850cf248f13d9da20c.html.

LA VANGUARDIA. (2016): "Manifestación en Barcelona por los derechos de los refugiados", 27 febrero 2016, https://www.lavanguardia.com/local/barcelona/20160227/4053391955/manifestacion-barcelona-refugiados.html.

LA VANGUARDIA. (2018): "España tiene pendiente acoger al 80\% de los refugiados que se comprometió a recibir", 11 noviembre 2018, https://www.lavanguardia.com/ politica/20181111/452848222139/espana-acoger-refugiados-comprometio-recibir. html.

MALM, S., E. O'FLYNN y H. ROBERTS. (2015): “Horror of trapped migrants' final moments: How 300 terrified people were locked in ship's hold as it capsized on its way to Europe - leaving up to 900 dead", en Daily Mail, 19 abril 2015. http://www. dailymail.co.uk/news/article-3045468/Up-700-feared-dead-migrant-boat-sinksLibya-newspaper.html\#ixzz4g7QLdv6C.

MCADAM, D. y W. SEWELL. (2001): "It's About Time: Temporality in the Study of Social Movements and Revolutions", en Silence and Voice in Contentious Politics, Cambridge, Cambridge University Press, pp. 89-125.

MUÑOZ MÁRQUEZ, L. M. (2011): "La agencia y la regulación de la cooperación al desarrollo de la Generalitat de Cataluña”, en Revista de Investigaciones Políticas y Sociológicas, 10(1), 2011, pp. 113-126.

PARRA, M. (2020): Migrar i resistir. Històries de l'últim èxode a Europa, Barcelona, Descontrol.

PISANI, F. (2011): "Barcelone veut réinventer la participation citoyenne", en Le Monde, 13 noviembre 2011, https://www.lemonde.fr/planete/article/2015/11/13/barcelone-ou-la-reinvention-permanente_4809189_3244.html.

PORTOS, M. (2019): "Keeping dissent alive under the Great recession: no-radicalisation and protest in Spain after the eventful 15M/indignados campaign", Acta Politica, 54(1), pp. 45-74.

PORTOS, M. (2021): Grievances and Public Protests: Political Mobilisation in Spain in the Age of Austerity, Londres, Palgrave Macmillan.

PORTOS, M. y T. CARVALHO (2019): “Alliance building and eventful protests: comparing Spanish and Portuguese trajectories under the Great Recession", Social Movement Studies. https://doi.org/10.1080/14742837.2019.1681957.

ROYO, S. (2014): "After austerity: lessons from the Spanish experience", en Elcano Royal Institute, WP 11/2014, http://www.realinstitutoelcano.org/wps/wcm/connect/ 8dc5a200456f1f1685d0e511646dbfa9/WP11-2014-Royo-After-austerity-lessonsfrom-the-Spanish-experience.pdf?MOD=AJPERES\&CACHEID=8dc5a200456f1f 1 685d0e511646dbfa9.

SAFONT, L. (2017): “'Queremos acoger” reclama un frente común en defensa de las personas migrantes”, en Público, 18 febrero 2017, http://www.publico.es/sociedad/ multitudinaria-marcha-barcelona-favor-acogida.html.

SNOW, D. A. y R. D. BENFORD. (1988): "Ideology, frame resonance, and participant mobilization”, Int. Soc. Mov. Res., 1, pp. 197-218.

TEJERINA, B. y PERUGORRÍA, I. (eds.). (2017): Crisis and Social Mobilization in Contemporary Spain. The 15M Movement, Abingdon, Routledge. 
THE GUARDIAN. (2017): "Protesters in Barcelona urge Spain to take in more refugees", en The Guardian, 18 febrero 2017. https://www.theguardian.com/world/2017/ feb/18/protesters-in-barcelona-urge-spain-to-take-in-more-refugees.

YATES, L. (2015): "Everyday politics, social practices and movement networks: daily life in Barcelona's social centres", The British Journal of Sociology, 66: 236-258.

ZAMPONI, L. (2017): "Practices of Solidarity: Direct Social Action, Politicisation and Refugee Solidarity Activism in Italy", Mondi Migranti, 3, pp. 97-117.

ZARAGOZA-CRISTIANI, J. (2016): Empowerment through migration control cooperation: the Spanish-Moroccan case, Tesis doctoral, European University Institute, Florencia. 